\title{
Concordance between genomic alterations assessed by next-generation sequencing in tumor tissue or circulating cell-free DNA
}

\author{
Young Kwang Chae ${ }^{1,2,3, *}$, Andrew A. Davis ${ }^{2, *}$, Benedito A. Carneiro ${ }^{1,2,3}$, Sunandana \\ Chandra ${ }^{1,2,3}$, Nisha Mohindra ${ }^{2,3}$, Aparna Kalyan ${ }^{1,2,3}$, Jason Kaplann ${ }^{1,2,3}$, Maria \\ Matsangou ${ }^{1,2,3}$, Sachin Pai ${ }^{1,3}$, Ricardo Costa ${ }^{1,3}$, Borko Jovanovic ${ }^{2,3}$, Massimo \\ Cristofanilli' ${ }^{1,2,3}$, Leonidas C. Platanias ${ }^{1,2,3,4}$, Francis J. Giles ${ }^{1,2,3}$ \\ ${ }^{1}$ Developmental Therapeutics Program of Division of Hematology Oncology, Northwestern University, Chicago, IL, USA \\ ${ }^{2}$ Department of Medicine, Northwestern University Feinberg School of Medicine, Chicago, IL, USA \\ ${ }^{3}$ Robert H. Lurie Comprehensive Cancer Center of Northwestern University, Chicago, IL, USA \\ ${ }^{4}$ Department of Medicine, Jesse Brown Veterans Affairs Medical Center, Chicago, IL, USA \\ *These authors contributed equally to this work
}

Correspondence to: Young Kwang Chae, email: young.chae@northwestern.edu

Keywords: next-generation sequencing, cell-free DNA, genomic alterations, metastatic disease, lung cancer

Received: April 14, 2016 Accepted: August 10, $2016 \quad$ Published: August 30, 2016

\section{ABSTRACT}

Genomic analysis of tumor tissue is the standard technique for identifying DNA alterations in malignancies. Genomic analysis of circulating tumor cell-free DNA (cfDNA) represents a relatively non-invasive method of assessing genomic alterations using peripheral blood. We compared the concordance of genomic alterations between cfDNA and tissue biopsies in this retrospective study. Twenty-eight patients with advanced solid tumors with paired next-generation sequencing tissue and cfDNA biopsies were identified. Sixty-five genes were common to both assays. Concordance was defined as the presence or absence of the identical genomic alteration(s) in a single gene on both molecular platforms. Including all aberrations, the average number of alterations per patient for tissue and cfDNA analysis was 4.82 and 2.96, respectively. When eliminating alterations not detectable in the cfDNA assay, mean number of alterations for tissue and cfDNA was 3.21 and 2.96, respectively. Overall, concordance was 91.9-93.9\%. However, the concordance rate decreased to $11.8-17.1 \%$ when considering only genes with reported genomic alterations in either assay. Over $\mathbf{5 0 \%}$ of mutations detected in either technique were not detected using the other biopsy technique, indicating a potential complementary role of each assay. Across 5 genes (TP53, EGFR, KRAS, APC, CDKN2A), sensitivity and specificity were $59.1 \%$ and $94.8 \%$, respectively. Potential explanations for the lack of concordance include differences in assay platform, spatial and temporal factors, tumor heterogeneity, interval treatment, subclones, and potential germline DNA contamination. These results highlight the importance of prospective studies to evaluate concordance of genomic findings between distinct platforms that ultimately may inform treatment decisions.

\section{INTRODUCTION}

A central goal of precision medicine in oncology is to target genomic alterations with novel therapeutic agents in a timely manner as the tumor genomic profile evolves. Currently, genomic analysis of tissue biopsy is accepted as the gold standard strategy for identifying DNA genomic alterations in tumors using next-generation sequencing (NGS) among other techniques. While tumor tissue will continue to remain the optimal technique to guide targeted treatments and to best understand tumor architecture and histology, limitations exist. In some circumstances, tissue biopsies carry risks and can be technically challenging to repeat. For instance, major complication rates with thoracic biopsies have been reported at $5.2 \%$ [1]. In other instances, such as 
metastatic disease, actionable molecular alterations may exist at multiple sites, not easily accessible via a single tissue biopsy. Therefore, developing non-invasive techniques for characterizing tumor mutations at baseline and dynamically during treatment may be necessary to assess tumor evolution, to monitor therapy response, and to personalize changes in treatment.

Tumor heterogeneity represents a major challenge to personalized anticancer therapy. Intratumor heterogeneity can result in missing critical DNA genomic alterations using conventional tumor biopsies and underestimation of the genomic variability within a tumor [2-5]. While tumor tissue-based biopsies are limited by spatial and temporal (e.g., potential need for multiple invasive biopsies) considerations, circulating tumor cell-free DNA (cfDNA) assays have emerged as a less invasive method of assessing tumor genomic alterations using peripheral blood [6, 7]. cfDNA quantity is on average higher in patients with cancer compared to controls, but varies considerably, and is thought to arise from apoptotic and necrotic cells [8]. In localized disease, the proportion of purified cfDNA in the blood is extremely low, which may limit utility in these patients. For advanced tumors, cfDNA is variable with some tumor types such as pancreatic, ovarian, colorectal, breast, bladder, esophageal, melanoma, and hepatocellular carcinoma expressing higher percentages of cfDNA while others, such as brain, renal, prostate, and thyroid cancers having detectable circulating DNA in less than $50 \%$ of patients [9]. One study with an estimated $95 \%$ of patients having advanced or metastatic disease reported $58 \%$ of patients having at least one detectable alteration, which increased to $65 \%$ when excluding glioblastoma [10]. In contrast, only 1 of $222(0.45 \%)$ healthy controls was found to have an alteration present. In addition, tumor purity based on non-cancerous cells in the tumor microenvironment may also complicate cfDNA assays [11]. Still, cfDNA has the potential to capture DNA alterations in the peripheral blood in a more dynamic manner in particular types of advanced tumors when feasibility of repeat tissue biopsies is limited.

Potential applications of cfDNA assays include early detection of metastatic disease and monitoring of minimum residual disease [12]. For example, detection of emerging EGFR mutations (e.g., deletions in exon 19 and L858R substitutions in exon 21) in patients with non-small cell lung cancer (NSCLC), can guide treatment with EGFR tyrosine kinase inhibitors (TKIs) [13, 14]. In many cases, tumor evolution results in secondary EGFR T790M mutations in exon 20 leading to resistance to EGFR TKIs [15]. cfDNA assays may have the potential to identify when these secondary resistance mutations arise in the peripheral blood prior to detection of clinical or radiological progression of disease. A recent prospective study indicates potential for detecting EGFR and KRAS mutations with $100 \%$ positive predictive value using plasma droplet digital PCR, which can be used to detect a small number of known mutation targets [16]. EGFR
T790M mutations were also examined with specificity of $63 \%$, possibly related to tumor heterogeneity and false-negative tissue genotyping. In addition, recent studies have suggested that detection of mutational burden can potentially help predict response to immunotherapies such as the checkpoint inhibitors targeting programmed death 1 (PD-1) and programmed death ligand 1 (PD-L1), raising another potential application of cfDNA analysis in parallel with genomic analysis of tissue biopsies [17-19].

High concordance has been reported between tumor tissue NGS and cfDNA in studies investigating the presence of $E G F R$ alterations in NSCLC, multiple genes in pancreaticobiliary cancers (KRAS, TP53, APC, FBXW7, $S M A D 4)$, exons $12-13$ of $K R A S$ in colorectal cancer, $B R A F$ V600E and KIT mutations in melanoma, and $B R A F$, $E G F R, K R A S$, and PIK3CA across a variety of advanced cancers [20-23]. These studies report high specificity and diagnostic accuracy as greater than 80-90\% compared to the gold standard of tissue-based NGS. However, in these studies, the reported values are based predominantly on not detecting DNA alterations in either assay (e.g., no mutations detected in EGFR in the same patient). This limits potential applicability for whether this technology can be used to detect early mutations in the peripheral blood. One study reported an average concordance of $85.9 \%$ in advanced cancers when including mutations that were both present and absent and $90 \%$ when limiting the sample to patients with stage II colorectal cancer [22]. Other work has reported high concordance for real-time polymerase methods to detect targeted $B R A F$ V600 mutations with rapid turn around time [24].

In early-stage disease, the low levels of cfDNA in peripheral blood may limit long-term clinical applications. In advanced cancers, current data are lacking that targeting cfDNA mutations in the peripheral blood improve patient outcomes. More clinical data are necessary to determine whether NGS data derived from cfDNA assays sufficiently correlate with that obtained from tissue biopsies to determine if and when cfDNA assays may be beneficial clinically. The utility may exist in detecting treatment response and resistance, as opposed to replacing tumor biopsy for initial treatment decision making [25].

The goal of the present study was to identify concordance of genomic alterations obtained from tissue biopsies and cfDNA analyses for patients with advanced malignancy. This is necessary in order to assess the fidelity of cfDNA as many genomic alterations contribute to tumor heterogeneity. It is also critical to understand whether this information may be useful in patients in whom tumor tissue is unavailable to support clinical treatment decisions based on emergence of genomic alterations that can predict resistance to treatment. To our knowledge, this is one of the most systematic analyses in terms of number of genes to examine concordance across DNA alterations as assessed in tissue-based NGS and cfDNA. 
Table 1: Characteristics of patients with both tissue and cell-free DNA NGS testing

\begin{tabular}{|c|c|c|}
\hline & Number & Percentage $(\%)$ \\
\hline \multicolumn{3}{|l|}{ Age (years) } \\
\hline Median & 65 & \\
\hline \multicolumn{3}{|l|}{ Sex } \\
\hline Male & 9 & 32.1 \\
\hline Female & 19 & 67.9 \\
\hline \multicolumn{3}{|l|}{ Type of cancer } \\
\hline Lung & 14 & 50.0 \\
\hline Ovarian & 3 & 10.7 \\
\hline Endometrial & 2 & 7.1 \\
\hline Thyroid & 2 & 7.1 \\
\hline Hepatocellular & 2 & 7.1 \\
\hline Unknown primary & 2 & 7.1 \\
\hline Cholangiocarcinoma & 1 & 3.6 \\
\hline Gastroesophageal junction & 1 & 3.6 \\
\hline Peritoneal carcinoma & 1 & 3.6 \\
\hline \multicolumn{3}{|l|}{ Pathologic stage } \\
\hline III & 2 & 7.1 \\
\hline IV & 26 & 92.9 \\
\hline History of prior cancers & 7 & 25.0 \\
\hline \multicolumn{3}{|l|}{ Smoking History } \\
\hline Current/Former & 16 & 57.1 \\
\hline Never & 12 & 42.9 \\
\hline \multicolumn{3}{|c|}{ Biopsy site corresponds to primary tumor } \\
\hline Yes & 12 & 42.9 \\
\hline No & 14 & 50.0 \\
\hline Unknown & 2 & 7.1 \\
\hline \multicolumn{3}{|c|}{ Interval between tissue and blood sample collection } \\
\hline$<90$ days & 14 & 50.0 \\
\hline$>90$ days & 14 & 50.0 \\
\hline
\end{tabular}

\section{RESULTS}

\section{Patient characteristics}

Fifty-four patients were identified retrospectively to have cfDNA testing performed by a single commercial NGS sequencing provider. Of these, 29 patients had matched tumor tissue biopsy and peripheral blood cfDNA genomic analyses. One patient was excluded for insufficient sample for tissue-based FoundationOne testing. Table 1 shows the patient characteristics of the 28 patients included in the study. There were 14 lung cancer (10 adenocarcinoma, 1 poorly differentiated NSCLC, 1 squamous, 1 small cell, 1 large cell neuroendocrine), 3 ovarian ( 2 serous, 1 clear cell), 2 endometrial (1 mucinous, 1 epitheloid), 2 thyroid (1 papillary, 1 poorly differentiated not otherwise specified), 2 hepatocellular (1 clear cell, 1 undifferentiated), 2 unknown primary, 1 cholangiocarcinoma (mucinous), 1 gastroesophageal junction adenocarcinoma, and 1 peritoneal adenocarcinoma (serous). Overall, 14 of 28 $(50 \%)$ patients had lung cancer and 26 of $28(93 \%)$ had stage IV disease. The median interval between collecting each paired tumor biopsy was 89 days [8-3,448 days]. Nine patients had no treatment between biopsy collections.

\section{Concordance of tumor biopsy and cfDNA genomic analyses}

Concordance between the two assays was $91.9 \%$ $(1672 / 1820$ genes) including all genes examined (SD $4.31 \%$ ) (Table 2). Concordance was similar for the 14 lung cancer patients $(91.1 \%)$ as compared to the 14 patients 
Table 2: Composite NGS data comparing tissue biopsy with cfDNA

\begin{tabular}{|l|c|c|}
\hline Average concordance of genomic analyses when DNA alterations are present or absent & $91.9 \%$ & $93.9 \%^{+}$ \\
\hline Percent of tissue alterations found in cfDNA & $20.7 \%$ & $31.1 \%^{+}$ \\
\hline Percent of cfDNA alterations found in tissue & $33.7 \%$ & $33.7 \%^{+}$ \\
\hline Mean and SD of genomic alterations in cfDNA & $2.96(3.01)$ & $2.96(3.01)^{+}$ \\
\hline Mean and SD of genomic alterations in tissue & $4.82(3.02)$ & $3.21(2.25)^{+}$ \\
\hline
\end{tabular}

Note: variants of unknown significance (VUS) included; cfDNA, cell-free DNA; SD, standard deviation.

+ : excludes variants within overlapping genes not tested by Guardant 360 .

Table 3: Concordance and partial concordance among only genes with genomic alterations in either assay

\begin{tabular}{|c|c|c|c|c|c|c|}
\hline & $\begin{array}{c}\text { Genes } \\
\text { with DNA } \\
\text { aberrations } \\
(N=170)\end{array}$ & $\begin{array}{c}\text { Genes } \\
\text { with DNA } \\
\text { aberrations }+ \\
(N=129)\end{array}$ & $\begin{array}{c}<90 \text { days } \\
\text { between biopsies } \\
(N=77)\end{array}$ & $\begin{array}{l}>90 \text { days } \\
\text { between } \\
\text { biopsies } \\
(N=93)\end{array}$ & $\begin{array}{c}<90 \text { days } \\
\text { between } \\
\text { biopsies }+ \\
(N=58)\end{array}$ & $\begin{array}{c}>90 \text { days } \\
\text { between } \\
\text { biopsies }+ \\
(N=71)\end{array}$ \\
\hline Concordance & $11.8 \%$ & $17.1 \%$ & $12.7 \%$ & $10.8 \%$ & $17.2 \%$ & $16.8 \%$ \\
\hline $\begin{array}{c}\text { Partial } \\
\text { Concordance }\end{array}$ & $4.7 \%$ & $4.7 \%$ & $1.3 \%$ & $7.5 \%$ & $1.7 \%$ & $7.0 \%$ \\
\hline
\end{tabular}

$N=$ number of unique genes with DNA alterations.

Note: variants of unknown significance (VUS) included.

+: excludes variants within overlapping genes not tested by Guardant 360 .

with non-lung cancers $(92.6 \%)$. When excluding particular alterations within overlapping genes not sequenced by Guardant 360 , concordance was $93.9 \%$. For this analysis, concordance was also similar when comparing lung cancer patients $(93.3 \%)$ to non-lung cancer patients $(94.5 \%)$. Concordance was high across all patients with range of $81.5 \%-100 \%$. One of $28(3.6 \%)$ had complete concordance at the patient level. This patient had no genomic alterations detected in either assay.

The remaining analyses were subset analyses, specifically examining concordance for genes with a genomic alteration present in one or both assays (i.e., excluding double negatives, wild type/wild type). Among the subset of genes with reported genomic alterations in either assay $(N=170)$, concordance between the two assays was $11.8 \%$ with a partial concordance of $4.7 \%$. The full and partial concordance values were $17.1 \%$ and $4.7 \%$, respectively, when only including alterations detectable in both assays. When excluding cfDNA biopsies without any alterations detected $(N=6)$, concordance and partial concordance were $19.0 \%$ and $5.2 \%$, respectively. No significant differences were found when stratifying the sample based on genes with complete exon sequencing or copy number variant (CNV). The gene level concordance for each individual patient ranged from 0 to $33.3 \%$ with partial concordance of 0 to $28.6 \%$. When only examining variants detectable in both assays, gene level concordance was 0 to $66.7 \%$ with partial concordance of 0 to $28.6 \%$. The sample was also analyzed based on collection interval between biopsies, less than 90 days versus greater than 90 days (Table 3 ). Concordance was $12.7 \%$ with a partial concordance of $1.3 \%$ for results of both tumor tissue and cfDNA less than 90 days apart $(N=77)$. For biopsies more than 90 days apart, concordance was $10.8 \%$ with a partial concordance of $7.5 \%(N=93)$. The trend was similar when only including alterations detectable in both assays. In addition, when examining concordance for patients with no treatment between biopsies $(N=9)$, no significant differences were noted in this preliminary analysis.

When considering the same genes and variants analyzed by both platforms, $31.1 \%$ of tissue-based mutations were detected via cfDNA. In addition, $33.7 \%$ of cfDNA mutations were detected via tissue-based sequencing. Figure 1 shows the landscape of DNA mutations found in the NGS platforms in each patient. Figure 2 is an oncoprint chart displaying the different potential outcomes observed for the 10 representative genes across all patients.

\section{Cell-free DNA percent}

For genes with identical sequencing mutations $(N=23)$, the percent or allele frequency of altered cfDNA was analyzed. The mean allele frequency of altered cfDNA in the peripheral blood for the subset of mutations found in both tissue and circulating blood was $16.8 \%$ (SD $27.4 \%$ ) [range $0.1 \%-81.0 \%$ ]. Overall, $65 \%$ of these identical mutations ( 15 of 23), were found to have 
Table 4: Sensitivity, specificity, and diagnostic accuracy across 5 genes

\begin{tabular}{|c|c|c|c|c|c|c|c|c|c|}
\hline & & \multicolumn{2}{|c|}{$\begin{array}{c}\text { Tissue } \\
\text { Mutations }\end{array}$} & \multirow{2}{*}{$\begin{array}{l}\text { Sensitivity } \\
\text { (\%) }\end{array}$} & \multirow{2}{*}{$\begin{array}{l}\text { Specificity } \\
\text { (\%) }\end{array}$} & \multirow{2}{*}{$\begin{array}{l}\text { PPV } \\
(\%)\end{array}$} & \multirow{2}{*}{$\begin{array}{l}\text { NPV } \\
(\%)\end{array}$} & \multirow{2}{*}{$\begin{array}{c}\text { Diagnostic } \\
\text { Accuracy } \\
(\%)\end{array}$} & \multirow{2}{*}{$\begin{array}{l}\text { Youden's J } \\
\text { index }\end{array}$} \\
\hline \multicolumn{2}{|c|}{ cfDNA mutations } & $(+)$ & $(-)$ & & & & & & \\
\hline \multirow[t]{2}{*}{ TP53 } & $(+)$ & 8 & 2 & & & & & & \\
\hline & $(-)$ & 2 & 14 & 80.0 & 87.5 & 80.0 & 87.5 & 78.6 & 0.7 \\
\hline \multirow[t]{2}{*}{$\overline{\text { EGFR }}$} & $(+)$ & 1 & 2 & & & & & & \\
\hline & $(-)$ & 1 & 24 & 50.0 & 92.3 & 33.3 & 96.0 & 89.3 & 0.4 \\
\hline \multirow[t]{2}{*}{ KRAS } & $(+)$ & 4 & 1 & & & & & & \\
\hline & $(-)$ & 2 & 21 & 66.7 & 95.5 & 80.0 & 91.3 & 89.3 & 0.6 \\
\hline \multirow[t]{2}{*}{ APC } & $(+)$ & 0 & 1 & & & & & & \\
\hline & $(-)$ & 4 & 23 & 0.0 & 95.8 & 0.0 & 85.2 & 82.1 & 0 \\
\hline \multirow[t]{2}{*}{ CDKN2A } & $(+)$ & 0 & 0 & & & & & & \\
\hline & $(-)$ & 0 & 28 & $\mathrm{n} / \mathrm{a}$ & 100.0 & $\mathrm{n} / \mathrm{a}$ & 100.0 & 100.0 & $\mathrm{n} / \mathrm{a}$ \\
\hline \multicolumn{2}{|c|}{ Total positive } & 13 & 6 & & & & & & \\
\hline \multicolumn{2}{|c|}{ Total negative } & 9 & 110 & & & & & & \\
\hline \multicolumn{2}{|c|}{$\begin{array}{l}\text { Total (positive }+ \\
\text { negative) }\end{array}$} & 22 & 116 & 59.1 & 94.8 & 68.4 & 92.4 & 89.1 & 0.5 \\
\hline
\end{tabular}

PPV: positive predictive value

NPV: negative predictive value

cfDNA: cell-free DNA

Note: variants of unknown significance (VUS) and variants not tested by Guardant360 excluded.

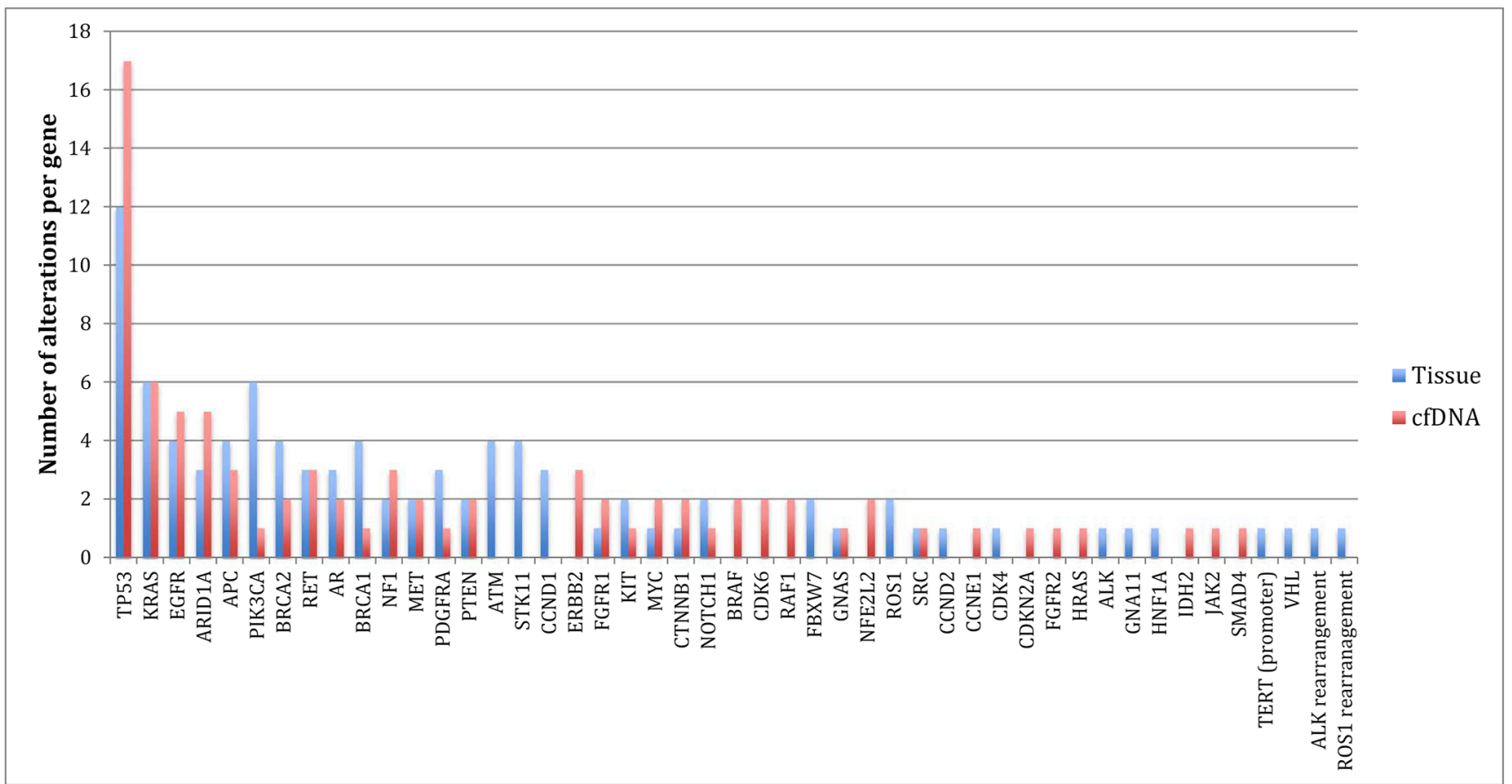

Note: excludes variants within overlapping genes not tested by Guardant360

Figure 1: Comparing frequency of alterations per gene in tissue and plasma cfDNA. 
relatively low (less than $10 \%$ ) allele frequency of altered circulating cfDNA.

\section{Average number of DNA alterations}

The average number of alterations including VUS per patient for tissue and cfDNA was 4.82 (SD 3.02) and 2.96 (SD 3.01), respectively (Table 2). Median number of mutations for tissue and cfDNA was 4 and 2, respectively. More mutations were detected in tissue-based NGS in 21 of $28(75 \%)$ of patients. A greater number of genomic alterations were detected in cfDNA in 5 of $28(17.9 \%)$ patients. An equal number of mutations were detected in 2 of $28(7.1 \%)$ patients. When excluding particular alterations within overlapping genes not sequenced by Guardant360, average number of alterations including VUS for tissue and cfDNA was 3.21 (SD 2.25) and 2.96 (SD 3.01). Median number of alterations was 3 for the tissue assay and 2 for the cfDNA assay.

\section{Diagnostic accuracy analysis}

Gene-level sensitivity, specificity, positive predictive value (PPV), negative predictive value (NPV) and diagnostic accuracy were analyzed across the five representative genes in the sample - TP53, EGFR, KRAS, $A P C, C D K N 2 A$ (Table 4). Tissue-based NGS was used as the gold standard for this analysis [26]. Across all 5 genes examined, including VUS, sensitivity was $50.0 \%$, specificity was $89.5 \%$, and diagnostic accuracy was $82.6 \%$ (Supplementary Table S3). Values were slightly higher when VUS were excluded with sensitivity of $59.1 \%$, specificity of $94.8 \%$, and diagnostic accuracy of $89.1 \%$ (Table 4). Four of the 5 genes examined had a specificity of greater than $90 \%$. Specificity and diagnostic accuracy were lowest for TP53. The Youden's J index across all 5 genes was 0.4 when including VUS and 0.5 when excluding VUS.

\section{DISCUSSION}

Personalized treatment in oncology aims to treat malignancies based on their genomic profile with effective molecularly targeted drugs. Additionally, the goal is to detect the emergence of genetic alterations that predict recurrence or resistance to treatment prior to development of clinical symptoms or radiological evidence of disease progression. Rapid developments in genomic analyses of tumors are enabling this transformative paradigm in oncology that may ultimately allow real-time treatment decisions based on the precise genomic landscape of the tumor. In certain circumstances, genomic analysis of cfDNA may hold promise to help overcome this challenge if findings reliably correlate with primary tumor and metastatic site(s) genomic landscape. Hence, the primary objective of this study was to investigate the concordance rate of genomic profiling using NGS in both tissue biopsies and peripheral blood circulating tumor cfDNA.

As compared to tissue-based biopsy, our findings indicate that cfDNA assays have high specificity, but low sensitivity, along with diagnostic accuracy in the range of $82-89 \%$. When examining all genes, including those without DNA alterations in either assay, concordance was 91.9-93.9\%. However, when examining the subset of genes with DNA alterations found in either assay, concordance and partial concordance were low (11.8-17.1\% and 4.7\%, respectively). A recent study examining 50 hotspot genes in tumor and cfDNA reported a sensitivity of $49.9 \%$ and a specificity of $99.8 \%$ for patients with advanced or metastatic solid tumors [27]. Similarly, previous studies that

\begin{tabular}{|l|l|l|l|l|l|l|l|l|l|l|l|l|l|l|l|l|l|l|l|l|l|l|l|l|l|l|l|l|}
\hline GenesPatients & 1 & 2 & 3 & 4 & 5 & 6 & 7 & 8 & 9 & 10 & 11 & 12 & 13 & 14 & 15 & 16 & 17 & 18 & 19 & 20 & 21 & 22 & 23 & 24 & 25 & 26 & 27 & 28 \\
\hline TP53 & & & & & & & & & & & & & & & & & & & & & & & & & & & & \\
\hline KRAS & & & & & & & & & & & & & & & & & & & & & & & & & & \\
\hline EGFR & & & & & & & & & & & & & & & & & & & & & & & & & \\
\hline ARID1A & & & & & & & & & & & & & & & & & & & & & & & & & & \\
\hline RET & & & & & & & & & & & & & & & & & & & & & & & & & & & \\
\hline APC & & & & & & & & & & & & & & & & & & & & & & & & & & \\
\hline CDKN2A & & & & & & & & & & & & & & & & & & & & & & & & & & \\
\hline PIK3CA & & & & & & & & & & & & & & & & & & & & & & & & & & & \\
\hline BRCA2 & & & & & & & & & & & & & & & & & & & & & & & & & & \\
\hline NF1 & & & & & & & & & & & & & & & & & & & & & & & & & & & \\
\hline
\end{tabular}

A total of 28 patients were tested for both NGS assays: tissue and plasma cfDNA.

Red: plasma mutation

Blue: tissue mutation

Green: concordant plasma and tissue mutation

Purple: partially concordant

Orange: plasma and tissue mutations present in the same gene, but discordant

White: no mutation present

Note: variants of unknown significance (VUS) included; variants within overlapping genes not tested by Guardant360 excluded

Figure 2: Oncoprint chart for 10 representative genes across all patients. 
report high concordance and specificity are based on single genes and sometimes specific codons. A critical difference in our analyses was that the genomic platforms used in our study included genes with both critical and complete exon coverage, thereby looking more comprehensively at sequencing concordance of the 65 overlapping genes. This analysis was critical to appropriately assess the potential for cfDNA to accurately identify specific sequencing mutations and resistance patterns. For example, it is not sufficient to classify a $B R A F$ V600E mutation in tissue and a $B R A F$ S729L VUS in peripheral blood as concordant at the gene level. Instead, we only considered concordant DNA alterations when the exact same sequencing alteration was present in both biopsies. Collectively, these data indicate that cfDNA assays may be best utilized to rule in rather than to rule out certain genomic alterations given the high specificity.

There are several potential reasons to explain the difference in results between the two NGS assays. First, the biopsy techniques are quite different. In lung cancer, previous studies using multi-region whole-exome and/or whole-genome sequencing indicate differing degrees of driver mutation heterogeneity with one study reporting 20/21 known cancer gene mutations in all regions [28]. However, another study suggested that subclonal diversification results in missed driver mutations indicating that multiple tissue biopsies in different locations would be optimal to best characterize tumor heterogeneity [29]. In contrast, cfDNA only captures mutations above the detection threshold that are found in peripheral blood after tumor cells outgrow the blood supply, become hypoxic, and undergo apoptosis or necrosis, releasing DNA into the peripheral blood. Therefore, there are intrinsic differences in assay platform and sensitivity. With current sequencing technology, more genomic alterations were detected in tissue biopsies (mean 4.82) as compared to cfDNA (mean 2.96). However, when eliminating alterations not detectable with the cfDNA at this time, mean alterations for tissue (3.21) and cfDNA (2.96) were similar. While our analyses compared a single, commercially available cfDNA assay to tissue-based NGS, the emergence of additional cfDNA platforms necessitates further studies comparing these to tissue-based NGS. Studies are ongoing to assess concordance using new platforms, as well as more sensitive droplet digital PCR for targeted sequencing alterations.

Second, temporal factors may also be significant. Tumors are highly dynamic and a larger sample size stratifying concordance based on timeframe between biopsies is critical. For patients with advanced cancer and sufficient cfDNA in the blood, we hypothesized that higher concordance would be associated with closer timeframe between biopsies. We were unable to validate this hypothesis based on our analysis (Table 3 ).

Third, type of cancer and site of biopsy are likely important. Some types of tumors, such as pancreatobiliary cancers, are more difficult to biopsy and to capture heterogeneity with tissue-based biopsies. Previous studies have also indicated differences in regard to type of malignancy and degree of cfDNA detected in the blood. Pancreatic, ovarian, colorectal, breast, bladder, gastroesophageal, melanoma, and hepatocellular carcinoma were more likely to have detectable cfDNA as compared to primary brain, renal, prostate, and thyroid cancers [9]. Our study indicated that concordance for patients with lung cancer was similar when compared to concordance for non-lung cancers. In addition, in our sample, $50 \%$ of tissue biopsies were performed at metastatic sites, which likely captures greater tumor heterogeneity after accumulation of mutations that promote metastasis. The burden and location of metastatic disease also may play a role in cfDNA detection and genomic landscape heterogeneity.

Finally, we included both subclones and VUS in this analysis with the goal of fully analyzing sequencing concordance between the two techniques. However, the potential to make treatment related decisions based on these assays is mostly predicated on clinically significant mutations at this time. Further comprehensive preclinical studies are needed to analyze this subset of mutations to determine the true functional significance of such variants. In addition, future studies should examine concordance comparing synonymous mutations across NGS platforms. Interestingly, over half of mutations detected in either technique were not detected using the other biopsy technique (Table 2, Supplementary Table S4). This finding indicates considerable tumor heterogeneity that cannot be fully detected in either biopsy technique alone. This implies a potential complementary role of both tissue-based and cfDNA biopsies to better capture tumor heterogeneity.

To the best of our knowledge, the study examines the concordance of the largest number of genes (65) to date and reflects what is found in a real-world oncology clinical setting. Our analyses were comprehensive in assessing concordance for the panel of overlapping genes tested in both NGS platforms: tissue and blood cfDNA. We defined concordance in our study to encompass the full set of genes tested, rather than focusing on each gene. We included VUS and subclones to fully capture sequencing concordance. Theoretically, there should be no difference in concordance between VUS and non-VUS. This is supported by similarities in our sensitivity, specificity, and diagnostic accuracy analyses with and without VUS (Table 4 and Supplementary Table S3). We included a heterogeneous group of cancers in our analysis to optimally examine concordance across multiple types of cancer. Limitations of our study include a relatively small patient sample size, and half of the sample consisting of patients with lung cancers, which may have skewed the most common genes encountered in our sample. A large prospective trial assessing concordance between circulating tumor DNA and FoundationOne matched solid tumor samples is currently underway (NCT02620527). 
In conclusion, our findings indicate high specificity and concordance when genomic alterations are present or absent. When examining the subset of genes with DNA alterations present, concordance was relatively low. Further studies are warranted to validate our findings across multiple cancer types, to examine concordance as new cfDNA platforms and sequencing technologies develop, to compare concordance at different intervals between biopsies, and to determine change in cfDNA genomic alteration type and frequency over time.

\section{MATERIALS AND METHODS}

\section{Study design and patients}

The Institutional Review Board of Northwestern University approved the study. All patients were recruited within the Northwestern Medicine Developmental Therapeutics Program, Division of Hematology Oncology, Northwestern University Feinberg School of Medicine. All studies were conducted in concordance with the Declaration of Helsinki. Fifty-four consecutive patients with commercial cfDNA NGS testing by Guardant360 (Guardant Health, Redwood City, CA) were identified retrospectively. Of these, 29 patients had comprehensive tissue NGS testing (FoundationOne) commercially performed by Foundation Medicine (Cambridge, MA) [26]. One patient was excluded because the FoundationOne report stated inadequate sample quality. Therefore, the final sample size was 28 patients with both tissue and peripheral blood cfDNA results available. Clinical characteristics of patients included in the study were retrospectively obtained via patient chart review. These data included basic demographics as well as tumor biopsy information to characterize the histology and stage of malignancy.

\section{Genes analyzed}

The study examined concordance between all genes found in cfDNA that were also present in tumor biopsy samples. In total, 68 genes were tested by the Guardant360 cfDNA. Of those, 3 genes were excluded (RHEB, RHOA, and RIT1) because these genes were not included in the 315 gene panel tested by tissue biopsy sequencing. Therefore, 65 genes common to both assays were examined for concordance (Supplementary Table S1). Twenty-nine genes had complete exon sequencing by both assays. Thirty-nine genes had critical exon sequencing by Guardant360 and complete exon sequencing by Foundation Medicine. Four rearrangements were common to both assays. The median interval between collections of tissue biopsy and peripheral blood specimens was 89 days.

\section{Defining concordance and data analysis}

Concordance analysis between genomic findings from tumor tissue biopsy and plasma cell-free DNA was performed on 65 genes. Two definitions of concordance were utilized in the study. First, concordance was defined at the gene level as detecting an identical sequencing mutation or not detecting an alteration in a single gene. For example, a R248L mutation in TP53 detected in both assays for the same patient was counted as a concordant genetic alteration. In contrast, the finding of distinct mutations detected in the same gene when the two assays were performed on the same patient was counted as a discordant genomic alteration (e.g., TP53 R248W by cfDNA and TP53 K132R by tissue biopsy) (Supplementary Table S2). For this analysis, the denominator in this calculation was 1,820 (65 genes for 28 patients).

Second, concordance was examined for the subset of genes in which a genomic alteration was found. For this analysis, genes in which mutations were not found (e.g., no mutations found in EGFR in both assays in the same patient) were excluded from both the numerator and denominator. Concordance was further compared when excluding particular alterations within overlapping genes not sequencing by Guardant360. These included splice site mutations, certain small insertions or deletions, and allelic loss (such as PTEN). Partial concordance was defined as having one concordant mutation and at least one discordant genomic alteration in the same gene. Alterations were binned into 3 categories: concordant, partially concordant, or discordant. Total concordance was defined, not by patients, but by the total number of fully concordant or partially concordant alterations with the denominator as the total number of DNA alterations in our sample $(N=170$ genomic alterations or $N=129$ when excluding alterations not sequenced by the cfDNA assay). The analysis included non-synonymous DNA mutations, rearrangements, and copy number variants (CNV) regardless of clone percentage. As variants of unknown significance (VUS) are also important to assess sequencing concordance, these were also included. All cfDNA samples, regardless of the number of DNA alterations detected (e.g., even when none were detected) were included. Synonymous DNA alterations reported by Guardant360 were not included in any concordance analysis because synonymous alterations were not included in FoundationOne reports.

In addition, sensitivity, specificity, and diagnostic accuracy (effectiveness) analyses were performed across the 5 representative genomic alterations (TP53, EGFR, $K R A S, A P C, C D K N 2 A$ ) in the sample (Figure 1). This analysis included instances in which no alterations were detected in either assay (double negatives). There were two instances for TP53 in which a DNA alteration was detected in the same gene in both assays, but the 
sequencing mutation was discordant. These two samples were excluded, only from the sensitivity, specificity, and diagnostic accuracy analysis for TP53 $(N=26)$. All other analyses included 28 patients. Youden's J index (sensitivity + specificity -1$)$ was calculated as an indirect measurement of concordance, as well as an alternative method reflecting diagnostic accuracy [30].

\section{ACKNOWLEDGMENTS AND FUNDING}

None.

\section{CONFLICTS OF INTEREST}

None.

\section{REFERENCES}

1. Overman MJ, Modak J, Kopetz S, Murthy R, Yao JC, Hicks ME, Abbruzzese JL, Tam AL. Use of research biopsies in clinical trials: are risks and benefits adequately discussed? J Clin Oncol. 2013; 31:17-22.

2. Gerlinger M, Rowan AJ, Horswell S, Larkin J, Endesfelder D, Gronroos E, Martinez P, Matthews N, Stewart A, Tarpey P, Varela I, Phillimore B, Begum S, et al. Intratumor heterogeneity and branched evolution revealed by multiregion sequencing. NEJM. 2012; 366:883-892.

3. Hiley C, de Bruin EC, McGranahan N, Swanton C. Deciphering intratumor heterogeneity and temporal acquisition of driver events to refine precision medicine. Genome Biology. 2014; 15:453.

4. Nik-Zainal S, Van Loo P, Wedge DC, Alexandrov LB, Greenman CD, Lau KW, Raine K, Jones D, Marshall J, Ramakrishna M, Shlien A, Cooke SL, Hinton J, et al. The life history of 21 breast cancers. Cell. 2012; 149:994-1007.

5. Wang Y, Waters J, Leung ML, Unruh A, Roh W, Shi X, Chen K, Scheet P, Vattathil S, Liang H, Multani A, Zhang H, Zhao R, et al. Clonal evolution in breast cancer revealed by single nucleus genome sequencing. Nature. 2014; 512:155-160.

6. De Mattos-Arruda L, Cortes J, Santarpia L, Vivancos A, Tabernero J, Reis-Filho JS, Seoane J. Circulating tumour cells and cell-free DNA as tools for managing breast cancer. Nat Rev Clin Oncol. 2013; 10:377-389.

7. Elshimali YI, Khaddour H, Sarkissyan M, Wu Y, Vadgama JV. The clinical utilization of circulating cell free DNA (CCFDNA) in blood of cancer patients. Int J Mol Sci. 2013; 14:18925-18958.

8. Jahr S, Hentze H, Englisch S, Hardt D, Fackelmayer FO, Hesch RD, Knippers R. DNA fragments in the blood plasma of cancer patients: quantitations and evidence for their origin from apoptotic and necrotic cells. Cancer Res. 2001; 61:1659-1665.
9. Bettegowda C, Sausen M, Leary RJ, Kinde I, Wang Y, Agrawal N, Bartlett BR, Wang H, Luber B, Alani RM, Antonarakis ES, Azad NS, Bardelli A, et al. Detection of circulating tumor DNA in early- and late-stage human malignancies. Sci Transl Med. 2014; 6:224ra224.

10. Schwaederle M, Husain H, Fanta PT, Piccioni DE, Kesari S, Schwab RB, Banks KC, Lanman RB, Talasaz A, Parker BA, Kurzrock R. Detection rate of actionable mutations in diverse cancers using a biopsy-free (blood) circulating tumor cell DNA assay. Oncotarget. 2016; 7:9707-9717. doi: 10.18632/oncotarget.7110.

11. Aran D, Sirota M, Butte AJ. Systematic pan-cancer analysis of tumour purity. Nat Commun. 2015; 6:8971.

12. Diaz LA, Jr., Bardelli A. Liquid biopsies: genotyping circulating tumor DNA. J Clin Oncol. 2014; 32:579-586.

13. Pao W, Miller V, Zakowski M, Doherty J, Politi K, Sarkaria I, Singh B, Heelan R, Rusch V, Fulton L, Mardis E, Kupfer D, Wilson R, et al. EGF receptor gene mutations are common in lung cancers from "never smokers" and are associated with sensitivity of tumors to gefitinib and erlotinib. PNAS. 2004; 101:13306-13311.

14. Mok TS, Wu YL, Thongprasert S, Yang CH, Chu DT, Saijo N, Sunpaweravong P, Han B, Margono B, Ichinose Y, Nishiwaki Y, Ohe Y, Yang JJ, et al. Gefitinib or carboplatinpaclitaxel in pulmonary adenocarcinoma. NEJM. 2009; 361:947-957.

15. Arcila ME, Oxnard GR, Nafa K, Riely GJ, Solomon SB, Zakowski MF, Kris MG, Pao W, Miller VA, Ladanyi M. Rebiopsy of lung cancer patients with acquired resistance to EGFR inhibitors and enhanced detection of the T790M mutation using a locked nucleic acid-based assay. Clin Cancer Res. 2011; 17:1169-1180.

16. Sacher AG, Paweletz C, Dahlberg SE, Alden RS, O'Connell A, Feeney N, Mach SL, Janne PA, Oxnard GR. Prospective Validation of Rapid Plasma Genotyping for the Detection of EGFR and KRAS Mutations in Advanced Lung Cancer. JAMA Oncol. 2016; 2:1014-1022.

17. Snyder A, Makarov V, Merghoub T, Yuan J, Zaretsky JM, Desrichard A, Walsh LA, Postow MA, Wong P, Ho TS, Hollmann TJ, Bruggeman C, Kannan K, et al. Genetic basis for clinical response to CTLA-4 blockade in melanoma. NEJM. 2014; 371:2189-2199.

18. Le DT, Uram JN, Wang H, Bartlett BR, Kemberling H, Eyring AD, Skora AD, Luber BS, Azad NS, Laheru D, Biedrzycki B, Donehower RC, Zaheer A, et al. PD-1 Blockade in Tumors with Mismatch-Repair Deficiency. NEJM. 2015; 372:2509-2520.

19. Birkbak NJ, Kochupurakkal B, Izarzugaza JM, Eklund AC, Li Y, Liu J, Szallasi Z, Matulonis UA, Richardson AL, Iglehart JD, Wang ZC. Tumor mutation burden forecasts outcome in ovarian cancer with BRCA1 or BRCA2 mutations. PloS One. 2013; 8:e80023. 
20. Mok T, Wu YL, Lee JS, Yu CJ, Sriuranpong V, SandovalTan J, Ladrera G, Thongprasert S, Srimuninnimit V, Liao M, Zhu Y, Zhou C, Fuerte F, et al. Detection and Dynamic Changes of EGFR Mutations from Circulating Tumor DNA as a Predictor of Survival Outcomes in NSCLC Patients Treated with First-line Intercalated Erlotinib and Chemotherapy. Clin Cancer Res. 2015; 21:3196-3203.

21. Zill OA, Greene C, Sebisanovic D, Siew LM, Leng J, Vu M, Hendifar AE, Wang Z, Atreya CE, Kelley RK, Van Loon K, Ko AH, Tempero MA, et al. Cell-Free DNA NextGeneration Sequencing in Pancreatobiliary Carcinomas. Cancer Discov. 2015; 5:1040-1048.

22. Kim ST, Lee WS, Lanman RB, Mortimer S, Zill OA, Kim KM, Jang KT, Kim SH, Park SH, Park JO, Park YS, Lim HY, Eltoukhy $\mathrm{H}$, et al. Prospective blinded study of somatic mutation detection in cell-free DNA utilizing a targeted 54-gene next generation sequencing panel in metastatic solid tumor patients. Oncotarget. 2015; 6:40360-40369. doi: 10.18632/oncotarget.5465.

23. Janku F, Angenendt P, Tsimberidou AM, Fu S, Naing A, Falchook GS, Hong DS, Holley VR, Cabrilo G, Wheler JJ, Piha-Paul SA, Zinner RG, Bedikian AY, et al. Actionable mutations in plasma cell-free DNA in patients with advanced cancers referred for experimental targeted therapies. Oncotarget. 2015; 6:12809-12821. doi: 10.18632/ oncotarget. 3373

24. Janku F, Claes B, Huang HJ, Falchook GS, Devogelaere B, Kockx M, Bempt IV, Reijans M, Naing A, Fu S, PihaPaul SA, Hong DS, Holley VR, et al. BRAF mutation testing with a rapid, fully integrated molecular diagnostics system. Oncotarget. 2015; 6:26886-26894. doi: 10.18632/ oncotarget.4723.
25. Williams PM, Conley BA. Clinical Application of Liquid Biopsies. JAMA Oncol. 2016; 2:1003-1005.

26. Frampton GM, Fichtenholtz A, Otto GA, Wang K, Downing SR, He J, Schnall-Levin M, White J, Sanford EM, An P, Sun J, Juhn F, Brennan K, et al. Development and validation of a clinical cancer genomic profiling test based on massively parallel DNA sequencing. Nat Biotechnol. 2013; 31:1023-1031.

27. Jovelet C, Ileana E, Le Deley MC, Motte N, Rosellini S, Romero A, Lefebvre C, Pedrero M, Pata-Merci N, Droin N, Deloger M, Massard C, Hollebecque A, et al. Circulating Cell-Free Tumor DNA Analysis of 50 Genes by NextGeneration Sequencing in the Prospective MOSCATO Trial. Clin Cancer Res. 2016; 22:2960-2968.

28. Zhang J, Fujimoto J, Zhang J, Wedge DC, Song X, Zhang J, Seth S, Chow CW, Cao Y, Gumbs C, Gold KA, Kalhor N, Little L, et al. Intratumor heterogeneity in localized lung adenocarcinomas delineated by multiregion sequencing. Science. 2014; 346:256-259.

29. De Bruin EC, McGranahan N, Mitter R, Salm M, Wedge DC, Yates L, Jamal-Hanjani M, Shafi S, Murugaesu N, Rowan AJ, Gronroos E, Muhammad MA, Horswell S, et al. Spatial and temporal diversity in genomic instability processes defines lung cancer evolution. Science. 2014; 346:251-256.

30. Youden WJ. Index for rating diagnostic tests. Cancer. 1950; $3: 32-35$. 